\title{
AVALIAÇÃO DE UM MÉTODO DE BAIXO CUSTO PARA DETERMINAÇÃO DA ATIVIDADE DA ALFA-AMILASE SALIVAR E ESTRESSE EM INDIVÍDUOS COM LÍQUEN PLANO BUCAL.
}

\author{
Daiane Monique Lira de França ${ }^{1}$; Ynara Bosco de Oliveira Lima-Arsati²; Juliana \\ Araújo da Silva Simoura ${ }^{3}$ e Valéria Souza Freitas ${ }^{4}$ \\ 1. Bolsista PROBIC/UEFS, Graduando em Odontologia, Universidade Estadual de Feira de Santana, e-mail: \\ dailirafranca@hotmail.com \\ 2. Orientador, Departamento de Ciências Biológicas, Universidade Estadual de Feira de Santana, e-mail: \\ ynara@uefs.br \\ 3. Mestre em Saúde Coletiva, Departamento de saúde, Universidade Estadual de Feira de Santana, e-mail: \\ julianasimoura.uefs@gmail.com \\ 4. Coordenadora do NUCAO, Departamento de Saúde, Universidade Estadual de Feira de Santana, e-mail: \\ valeriauefs@gmail.com
}

PALAVRAS-CHAVE: saliva; estresse; alfa-amilase salivar.

\section{INTRODUÇÃO}

A alfa amilase salivar (sAA) é um dos componentes mais abundantes na saliva humana, representando 10-20\% do teor total de proteínas (Baum, 1993). Além de ser reconhecida por contribuir na digestão de amido e modular a adesão de bactérias às superfícies orais, a sAA também tem sido estudada como biomarcador de estresse (Nater \& Rohleder, 2009; Castilho et al. 2011).

Alguns autores reportaram o aumento da atividade de sAA sob o estresse (Nater et al., 2006). Diante de evidências de fatores psicológicos como o estresse na etiologia do líquen plano bucal (LPB), a busca por esses biomarcadores tem se tornado cada vez mais importante (Rödström et al., 2001; Koray et al., 2003; Shah, Ashok, Sujatha, 2009; Girardi et al., 2011; Nadendla et al., 2014; Pippi et al., 2014). Entretanto, apenas um estudo, até o momento, avaliou a sAA para a mensuração do estresse em indivíduos com esta doença, utilizando para tal um kit salivar de alto custo no mercado para dosagem da atividade deste biomarcador (Pippi et al., 2014).

Assim, apesar da quantificação de sAA em amostras de saliva utilizar uma técnica relativamente simples, as dosagens do padrão de secreção deste biomarcador utilizando kits de valor bastante elevado tem se tornado um obstáculo para a realização de estudos epidemiológicos com elevado tamanho amostral. Desta forma, considerando a disponibilidade comercial de kits de valor mais acessível e a importância do estudo de biomarcadores salivares como a sAA para a avaliação do estresse, o objetivo do presente trabalho será avaliar por meio de um método de baixo custo a atividade da alfa-amilase salivar e sua relação com o estresse em indivíduos com líquen plano bucal.

\section{MATERIAL E MÉTODOS OU METODOLOGIA (ou equivalente)}

Trata-se de um estudo clínico do tipo caso-controle. A população do estudo é constituída por casos diagnosticados com lesão de Líquen Plano Bucal LPB no CRLBNUCAO-UEFS e um número igual de controles, emparelhados por sexo e idade em relação aos indivíduos com a doença. A amostra do estudo foi composta por 16 indivíduos sendo $87,5 \%$ mulheres, com $81,25 \%$ apresentando idade acima dos 40 anos, média da idade 47,56 anos, desvio padrão de 9,71 anos e mediana de 49 anos.

Para avaliação dos escores de estresse foi aplicado um formulário de entrevista, a Escala de Estresse Percebido. Os pacientes foram instruídos a coletar a saliva em seus domicílios em Salivettes ${ }^{\circledR}$ (Sarstedst, Alemanha), devidamente identificados. A determinação da atividade da sAA, foi realizada pelo método colorimétrico de CARAWAY modificado. O presente projeto de pesquisa foi aprovado pelo Comitê de Ética em Pesquisa em Seres 
Humanos da Universidade Estadual de Feira de Santana (UEFS), segundo o CAAE: 05590612.7.0000.0053 e número de parecer 114.132.

RESULTADOS E/OU DISCUSSÃO (ou Análise e discussão dos resultados)

Ao empregar a Escala de Estresse Percebido (EPP), os escores variaram de 27 a 41 pontos, com mediana de 30 no grupo caso, e de 06 a 33 pontos, com mediana de 14,5 no grupo controle. Houve diferença estatisticamente significativa entre os grupos (teste de MannWhitney; $\mathrm{p}=0,0054)$, indicando uma associação entre a exposição ao estresse e a manifestação clínica do LPB o que foi apresentado também em outros estudos (Pourshahidi, Ebrahimi, Andisheh Tadbir, 2011; Valter et al., 2013; Gavic et al., 2014; Gholizadeh et al., 2014; Čanković, Bokor-Bratić, Novović, 2015) .

$\mathrm{Na}$ avaliação da concentração de sAA a tabela 1 apresenta a média e o desvio padrão encontrados em cada tempo de coleta. Pode se perceber que apenas ao acordar (T1) foi observada diferença estatisticamente significativa entre os grupos $(\mathrm{p}=0,016)$, sendo os maiores valores para o grupo controle.

O protocolo utilizado na avaliação da concentração de sAA em U/ml nas amostras desse estudo apresentou valores absolutos muito discrepantes dos apresentados em outros estudos. Enquanto no presente estudo a concentração de sAA em U/ml nas amostras são grandezas em dezenas de milhar, conforme aponta a tabela 1, a literatura exibe resultados em no máximo centenas (Nater et al., 2007; Rohleder, Nater, 2009; Maruyama et al., 2012; Veen et al., 2013).

Tabela 1. Comparação da média dos níveis de sAA (U/ml) entre casos de LPB e controles saudáveis ao acordar, trinta minutos após acordar e antes de dormir.

\begin{tabular}{c|ccc}
\hline & CASO & CONTROLE & $\mathrm{p}^{*}$ \\
\hline Ao acordar (T1) & $25122,60 \pm 206,79$ & $26960,90 \pm 1590,17$ & 0,016 \\
30 minutos após acordar & $25894,75 \pm 1044,13$ & $26238,97 \pm 1087,18$ & 0,543 \\
(T2) & $25648,45 \pm 1040,78$ & $26292,42 \pm 1338,623352$ & 0,300 \\
Antes de dormir (T3) & $2,60 \pm 4,50$ & $-14,20 \pm 34,84938773$ & 0,268 \\
\% T2 - T1 & $-0,90 \pm 3,53$ & $-0,32 \pm 3,613778725$ & 0,760 \\
M T3 - T2 & & & \\
Média de T1, T2 e T3 & $25605,61 \pm 675,40$ & $26557,71 \pm 1214,383594$ & 0,073 \\
\hline
\end{tabular}

*valores de $\mathrm{p} \leq 0,05$ indicam diferença estatisticamente significativa no teste $\mathrm{t}$. 


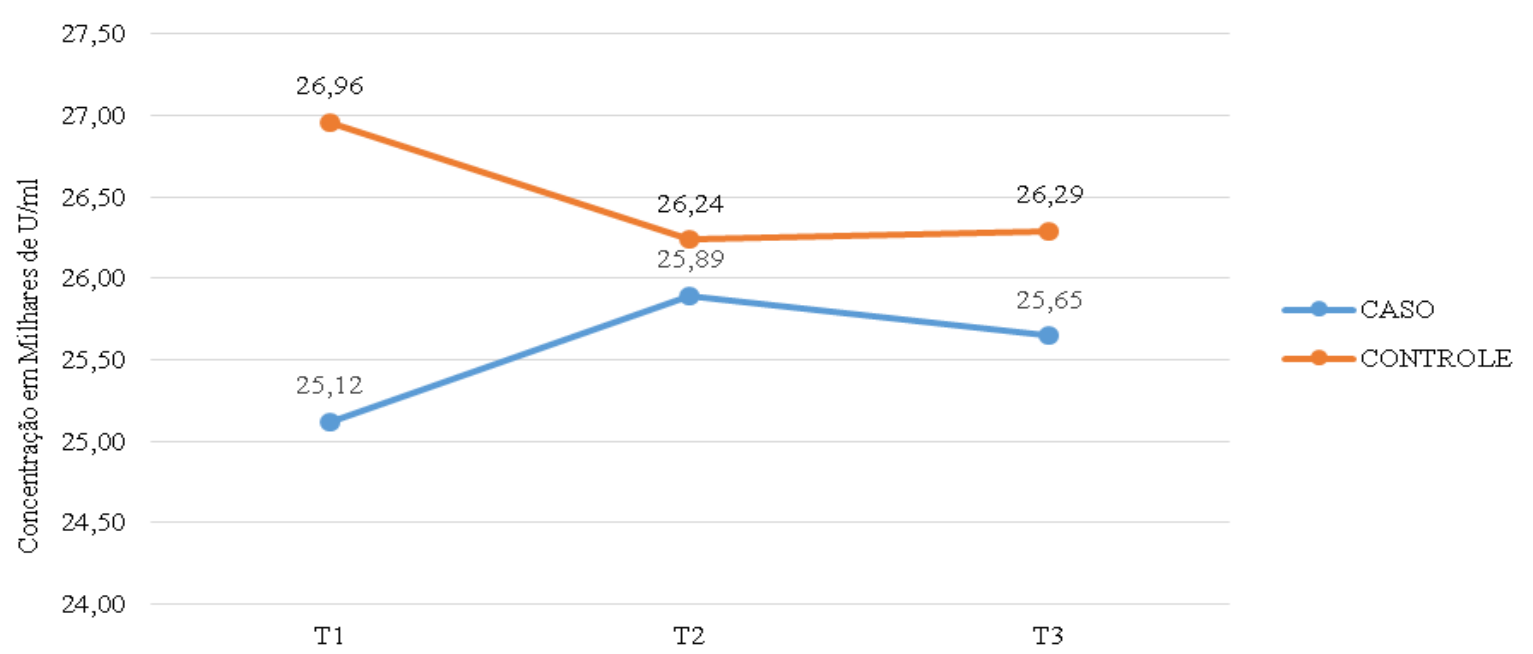

Figura 1: níveis médios de concentração de sAA ao acordar (T1), 30 após acordar (T2) e antes de dormir (T3) dos indivíduos sem LPB (controle) e com LPB (caso).

O perfil diurno da sAA é representado no gráfico 1 e mostra que nos casos ocorreu um aumento da concentração de alfa-amilase salivar nos primeiros 30 minutos após acordar como sugerido nos dados apresentados na tabela 1 onde a média do percentual de T2-T3 apresenta valor positivo, já a concentração de alfa amilase salivar nos controles nos 30 minutos após acordar apresentou um declínio e média do percentual de T2-T3 apresentando valor negativo. É observado uma diminuição da concentração em T3 em relação a T2 nos casos e um aumento nos controles. Entretanto não houve diferença estatisticamente significativa das concentrações de sAA no intervalo das coletas entre os grupos o que está de acordo com o estudo de Pippi et al. (2014).

Alguns estudos descrevem o padrão de secreção diária de sAA com o mesmo comportamento observado no grupo controle do presente estudo, ou seja, uma diminuição acentuada é normalmente observada até 30 a 60 minutos após acordar seguido por um aumento constante ao longo do dia (Nater et al., 2007). Outros estudos observaram um aumento constante da atividade de sAA ao longo do dia, com menores níveis observados ao acordar (Rohleder et al., 2004).

O coeficiente de correlação de Pearson (CCP) foi utilizado para verificar a correlação entre as concentrações de sAA e o escore da Escala de Estresse Percebido. Foi observada correlação negativa baixa e sem diferença estatisticamente para casos $(\mathrm{CCP}=-0,27$ com $\mathrm{p}=$ $0.52)$ e também para controles $(\mathrm{CCP}=-0,28$ com $\mathrm{p}=0,49)$. Outros estudo concordam com esse resultado pois não encontraram associação entre estresse percebido e atividade de sAA ( Wingenfeld et al., 2010; Castilho et al., 2011).

\section{CONSIDERAÇÕES FINAIS (ou Conclusão)}

Houve associação entre a exposição ao estresse e a manifestação clínica do LPB. Na avaliação da concentração de sAA (U/ml) entre casos de LPB e controles saudáveis ao acordar, trinta minutos após acordar e antes de dormir, apenas ao acordar pode se observar uma diferença estatisticamente significativa entre os grupos $(\mathrm{p}=0,016)$, sendo maior para indivíduos sem LPB. Não houve correlação entre estresse percebido e níveis de sAA, na amostra estudada. O protocolo utilizado na avaliação da concentração de sAA em U/ml nas amostras desse estudo, que é com reagentes de menor custo, apresentou valores absolutos muito discrepantes em comparação com a literatura. Isso pode indicar baixa sensibilidade e confiabilidade do método.

\section{REFERÊNCIAS}

BAUM, B.J. 1993. Principios of saliva secretion. Ann N Y Acad Sci, v.694,p. 17-23.

ČANKOVIĆ, M.; BOKOR-BRATIĆ, M.; NOVOVIĆ, Z. 2015. Stressful Life Events and Personality Traits in Patients with Oral Lichen Planus. Acta dermatovenerologica Croatica: $A D C$, v. 23, n. 4, p. 270-6. 
CASTILHO, M. C. DE M. et al. 2011. Relação entre estresse percebido e fatores salivares, em mulheres, sob condições basais de estresse. Arquivos em Odontologia, v. 47, n. 1, p. 2530 .

GAVIC, L. et al. 2014. The role of anxiety, depression, and psychological stress on the clinical status of recurrent aphthous stomatitis and oral lichen planus. Journal of oral pathology \& medicine: official publication of the International Association of Oral Pathologists and the American Academy of Oral Pathology, v. 43, n. 6, p. 410-7, jul.

GHOLIZADEH, N. et al. 2014. Evaluation of the serum zinc level in erosive and non-erosive oral lichen planus. Journal of dentistry (Shiraz, Iran), v. 15, n. 2, p. 52-6, jun.

GIRARDI, C. et al. 2011. Salivary cortisol and dehydroepiandrosterone (DHEA) levels, psychological factors in patients with oral lichen planus. Archives of oral biology, v. 56, n. 9, p. 864-8, set.

KORAY, M. et al. 2003. The evaluation of anxiety and salivary cortisol levels in patients with oral lichen planus. Oral diseases, v. 9, n. 6, p. 298-301.

MARUYAMA, Y. et al. 2012. Differences in salivary alpha-amylase and cortisol responsiveness following exposure to electrical stimulation versus the trier social stress tests. PLOS ONE.

NADENDLA et al. 2014. Salivary Cortisol and Anxiety levels in lichen planus Patients. Journal of Clinical and Diagnostic Research, v. 8, n. 12,p. ZC01-ZC03.

NATER, U. M. et al. 2007. Determinants of the diurnal course of salivary alpha-amylase. Psychoneuroendocrinology, v. 32, n. 4, p. 392-401, maio.

NATER, U. M. et al. 2006. Stress-induced changes in human salivary alpha-amylase activityassociations with adrenergic activity. Psychoneuroendocrinology, v. 31, p. 49-58.

NATER, U. M.; ROHLEDER, N. 2009. Salivary alpha-amylase as a non-invasive biomarker for the sympathetic nervous system: current state of research. Psychoneuroendocrinology, v. 34, n. 4, p. 486-96, maio.

PIPPI, R. et al. 2014. Diurnal trajectories of salivary cortisol, salivary $\alpha$-amylase and psychological profiles in oral lichen planus patients. Journal of biological regulators and homeostatic agents, v. 28, n. 1, p. 147-156.

POURSHAHIDI, S.; EBRAHIMI, H.; ANDISHEH TADBIR, A. 2011. Evaluation of the Relationship between Oral Lichen Planus and Stress. Journal of Dentistry, v. 12, n. 1, p. 43 47.

RÖDSTRÖM, P.O et al. 2001. Erosive oral lichen planus and saliva cortisol. J Oral Pathol Med., v.30, n.5, p. 257-263.

ROHLEDER, N. et al. 2004. Psychosocial stress-induced activation of salivary alphaamylase: an indicator of sympathetic activity? Annals of the New York Academy of Sciences, v. 1032, p. 258-63, dez.

ROHLEDER, N.; NATER, U. M. 2009. Determinants of salivary-amylase in humans and methodological considerations. Psychoneuroendocrinology.

SHAH B, ASHOK L, SUJATHA G P. 2015. Evaluation of salivary cortisol and psychological factors in patients with oral lichen planus. Indian J Dent Res. v.20, n. 3, p. 288-292.

VALTER, K. et al. 2013. The influence of psychological state on oral lichen planus. Acta clinica Croatica, v. 52, n. 2, p. 145-9, jun.

VEEN, G. et al. 2013. Evening salivary alpha-amylase, major depressive disorder, and antidepressant use in the Netherlands Study of Depression and Anxiety (NESDA). Psychiatry Research, v. 208, n. 1, p. 41-46.

WINGENFELD, K. et al. 2010. The diurnal course of salivary alpha-amylase in nurses: An investigation of potential confounders and associations with stress. Biological Psychology, v. 85 , n. 1, p. $179-181$. 